\title{
Fomentar la cultura preventiva entre trabajadores y empresarios
}

\section{Preventive promote culture between workers and employers}

\section{José Manuel González Tizón', Lucía Barandela Domuro'}

1. Asociacion empresarios del poligono industrial San Cibrao de Viñas.

Ourense. España.

\section{Correspondencia}

Lucía Barandela Domuro.

Polígono San Cibrao. Ourense. España.

Correo electrónico: lucia .barandela@poligonosancibrao.com

\section{ResUmen}

Desde hace más de una década, la entidad trabaja activamente en la puesta en marcha de acciones preventivas que mejoren las condiciones de seguridad y salud de los trabajadores y empresarios de la zona.

Palabras clave: Fomento, extinción, divulgación, apoyo.

\section{Abstract}

For more than a decade, the company is active in the implementation of preventive actions to improve the safety and health of workers and employers in the area.

Key words: promotion, extinction, reléase, support. 


\section{INTRODUCCIÓN}

La Asociación de Empresarios Polígono San Cibrao nace en 1979 con el objetivo de defender los intereses de las empresas que forman el área industrial de San Cibrao das Viñas, en la provincia de Ourense. Las más de 300 empresas, 8.000 trabajadores, 5.000.000 de metros cuadrados y 20.000 vehículos que transitan diariamente por las calles de esta zona industrial, le confieren a esta entidad una gran capacidad administrativa. La realización de un plan de buenas prácticas en materia de prevención de riesgos laborales a lo largo de los últimos 11 años, ha supuesto la reducción notable de los accidentes laborales en la zona y el fomento de la cultura preventiva entre trabajadores y empresarios.

La Asociación de Empresarios Polígono San Cibrao creó en 2002 el Servicio de Primera Intervención, ante la inseguridad y falta de equipamientos para hacer frente a un incendio. Este servicio consiste en un equipo de 10 profesionales en la extinción de incendios que atienden, en horario ininterrumpido de 24 horas los 365 días del año, al colectivo industrial no sólo de San Cibrao, sino también de todo el entorno que rodea a la zona empresarial formado por más de 40.000 habitantes. Este servicio cuenta con material necesario para resolver de forma rápida y eficaz cualquier emergencia: una bomba urbana ligera con equipo de excarcelación y bomba de presión combinada, vehículo de intervención rápida con bomba de alta presión y equipo de excarcelación, remolque multiservicio con material de rescate en altura, material de extinción de incendios, equipos de respiración autónoma, cámara térmica, y detector de gases y mangueras extintores, entre otro.

En este ámbito, el Servicio de Primera Intervención desarrolla una campaña informativa y de promoción de simulacros de emergencia en las empresas, totalmente gratuita para sus asociados y con el doble objetivo de formar a todo el personal en las rutinas de acción, para afrontar una situación de peligro y cumplir con la normativa básica de autoprotección.

La labor de este servicio es coordinada por un Gabinete de prevención de riesgos y seguridad, creado por la Asociación para sensibilizar y formar en materia de seguridad y salud laboral al tejido empresarial del polígono.

En el terreno formativo, el Gabinete orienta y conciencia a los alumnos de los riesgos que se dan en los puestos de trabajo, así como las medidas que deben llevar a cabo para prevenirlos. Acciones como el uso de extintores, el desarrollo práctico de planes de emergencia en las empresas, primeros auxilios y marcado CE en maquinaria, fueron algunos de los cursos impartidos, en los que se vieron superadas las expectativas de implicación empresarial.

Otra de las labores del Gabinete fue la realización de un Estudio de Planes de Emergencia Global del Polígono de San Cibrao, en el que se examinaron los riesgos de incendio, explosión, vertidos y fuga de gases de todas las empresas; y por otro lado un Análisis de las actividades de prevención de las empresas de la zona industrial, en el que se estudiaron los medios y recursos con los que cuentan las empresas para llevar a cabo su prevención de riesgos interna.

Ambos estudios sirvieron como base para realizar el Mapa de Riesgos, una aplicación gráfica del Polígono San Cibrao a través de la cual las empresas de esta área industrial podrán visualizar claramente los principales riesgos y situaciones de emergencia que se producen en la industria (riesgos de incendio, fugas de gases, vertidos peligrosos y explosiones) no sólo de su empresa, sino también de las empresas colindantes. La aplicación permite también la localización de bocas de incendio que servirán para que los servicios de emergencias, de San Cibrao y de toda la provincia, conozcan el estado de nuestro polígono y actúen de forma rápida y eficaz ante cualquier emergencia.

En el ámbito de la orientación y concienciación a las empresas y trabajadores del Polígono San Cibrao, se encuentran iniciativas como la entrega de boletines de actualidad preventiva "Infopoligono", que ponen de manifiesto todas las actividades desarrolladas 
por la Asociación, así como legislación actual de importancia para la industria y otras noticias de interés preventivo, acompañados de consejos prácticos, formación en prevención y entrevistas a personalidades de interés para la provincia de Ourense.

Destacamos además otras labores como la puesta en marcha del Plan de buenas prácticas y señalización, a través de la entrega de carteles informativos gráficos; proyectos de implantación de la certificación de calidad OHSAS 18001 entre las empresas de la zona industrial de San Cibrao; revisión y mantenimiento de todas las bocas de incendio que se distribuyen por el Polígono de San Cibrao.

Con el objetivo de continuar ofreciendo asesoramiento a las empresas y trabajadores, se ha puesto en marcha una campaña informativa, "Cero Accidentes, Cero Incidentes" a través de la cual se desarrollarán diferentes acciones encaminadas a reducir la siniestralidad laboral en nuestras empresas en los próximos cinco años: "Objetivo Cero Accidentes".

Dentro de esta acción se enmarcan las siguientes campañas informativas: Campaña de prevención de accidentes in itínere, con el objetivo de formar a empresarios y trabajadores en las buenas actitudes al volante y Campaña de prevención de riesgos cardiovasculares, con consejos prácticos de hábitos saludables.

Estas actividades cuentan con el soporte de una página web completa en la que el Gabinete pone a disposición de los usuarios la información relativa a actividades y consejos útiles en materia preventiva, en donde se podrán consultar los boletines informativos publicados, que también se edita en formato digital, con el objeto de dar mayor difusión y llegar a toda la sociedad.

El Gabinete de prevención continúa su andadura, mejorando los servicios en materia preventiva. Para ello ha implantado el uso de las nuevas tecnologías en este campo a través de aplicaciones informáticas para dispositivos iPad del mapa de riesgos o la creación de un blog en donde el público podrá expresar sus opiniones sobre cuestiones preventivas.

\section{RESULTADOS Y BENEFICIOS OBTENIDOS}

Todos estos servicios han mejorado, no sólo la seguridad empresarial, sino la calidad de vida del entorno municipal que rodea el polígono, ya que se cuentan con mayores dotaciones e infraestructuras que minimizan y evitan accidentes.

La Asociación, como única entidad gallega premiada por la Fundación para la prevención de riesgos laborales en el año 2009 y la revista "Formación de seguridad laboral" en 2011, se consolida como un referente a nivel nacional, al ser capaz de combinar la mejora permanente de la eficiencia empresarial con las técnicas de seguridad y salud laboral, poniendo todos los medios para reducir los accidentes y creando un ambiente propicio para la implantación de sistemas productivos seguros.

\section{AGRADECIMIENTOS}

Xunta de Galicia.

Consellería de Traballo e Benestar.

Diputación Provincial de Ourense.

Ayuntamiento de San Cibrao das Viñas. 